\title{
Tecnología lítica en el sitio Las Toscas 3 (llanura Interserrana, región pampeana, Argentina). Uso de materias primas líticas y manufactura de artefactos
}

\author{
Rocío Torino \\ Recibido 29 de abril 2019. Aceptado 07 de noviembre 2019
}

\begin{abstract}
RESUMEN
En este artículo se presentan y discuten los resultados del estudio de la tecnología lítica del sitio arqueológico superficial Las Toscas 3, ubicado en la Ilanura Interserrana en la subregión Pampa Húmeda (Argentina). El conjunto arqueológico recuperado está integrado mayormente por artefactos líticos, escasos restos faunísticos y fragmentos de pigmento mineral, resultantes de ocupaciones cazadoras-recolectoras en las márgenes de una laguna. El hallazgo de una punta de proyectil pequeña permite asignar parte de los materiales al Holoceno tardío (ca. 3000 años ${ }^{14} \mathrm{C}$ AP), aunque no es posible descartar ocupaciones previas. El objetivo que guio este trabajo fue determinar el modo de explotación de las diferentes materias primas y las técnicas empleadas para la producción de los instrumentos. Con este propósito, se presentan los resultados del análisis tecnomorfológico de los artefactos líticos, se analizan las estrategias de aprovisionamiento y se describen las cadenas operativas para cada una de las materias primas. Los resultados alcanzados indican que en el sitio se desarrollaron diversas tareas tecnológicas, con la ortocuarcita del Grupo Sierras Bayas como la roca mayormente explotada. Si bien se observa la reducción de núcleos con el objetivo de obtener soportes para la confección de una amplia variedad de instrumentos, se encuentran representadas, preferentemente, las etapas intermedias y finales de las cadenas operativas.
\end{abstract}

Palabras clave: Cazadores-recolectores; Rocas; Elecciones técnicas; Aprovisionamiento lítico; Cadenas operativas.

\begin{abstract}
LITHIC TECHNOLOGY FROM LAS TOSCAS 3 SITE, PAMPEAN INTERSERRANA GRASSLANDS, ARGENTINA: USE OF LITHIC RAW MATERIALS AND ARTIFACT MANUFACTURE. In this article, the results of the study of the lithic technology of Las Toscas 3 archaeological site, located in the Interserrana grasslands in the humid Pampa subregion, Argentina, are presented and discussed. The archaeological assemblage is composed mainly of lithic artifacts, scarce faunal remains, and fragments of mineral pigment, resulting from hunter-gatherer occupations on the banks of a shallow lake. The finding of a small projectile tip allow part of the materials to be assigned to the late Holocene (c. $3000 \mathrm{BP}$ ), although it is not possible to rule out previous occupations. The main goal that guided this research was to establish how different lithic raw materials were exploited and to study the techniques used in artifact production. To this end, we present the results of the techno-morphological analysis of the lithic artifacts, the analysis of procurement strategies, and a description of the operating chain for each raw material. The results achieved indicate that various technological tasks were developed at the site, with the Group Sierras Bayas orthoquarzite being the most exploited rock. Although the reduction of cores to obtain blanks for the manufacture of a wide variety of tools is observed, the intermediate and final stages of the operating chain are preferably represented.
\end{abstract}

Keywords: Hunter-gatherers; Rocks; Technical choices; Lithic provisioning; Operating chain.

Rocío Torino. Facultad de Ciencias Sociales, Universidad Nacional del Centro de la Provincia de Buenos Aires. Avda. Del Valle 5737. Agencia Nacional de Promoción Científica y Tecnológica (ANPCyT). División Arqueología, Facultad de Ciencias Naturales y Museo, Universidad Nacional de La Plata. Paseo del Bosque s/n (1900), La Plata. E-mail: florenciatorino@gmail.com 


\section{INTRODUCCIÓN. EL PANORAMA REGIONAL DURANTE EL HOLOCENO}

La subregión Pampa Húmeda presenta una distribución heterogénea de las materias primas líticas. Las fuentes de rocas que fueron utilizadas por los grupos humanos desde el Pleistoceno final son de tipo primario, como es el caso de los sistemas serranos de Tandilia y Ventania o pequeños afloramientos en el interior de la llanura Interserrana (Flegenheimer, 1991; Flegenheimer et al., 1996, 1999; Oliva y Moirano, 1997; Bayón et al., 1999; Bayón y Flegenheimer, 2004; Messineo et al., 2004; Catella et al., 2010; Colombo, 2011), o bien de tipo secundario, como los bancos de rodados costeros y fluviales (Bayón y Zavala, 1997; Bonomo, 2002, 2005; Aldazábal y Eugenio, 2010).

Durante el Holoceno, las sociedades cazadorasrecolectoras utilizaron diversas estrategias tecnológicas con respecto a la adquisición, transporte y uso de las materias primas líticas. Esta situación pudo deberse, por un lado, a la movilidad de los grupos humanos y a las distancias existentes entre los lugares de uso de los instrumentos líticos y las fuentes de abastecimiento de las diferentes litologías; por otro lado, a factores de índole social, simbólico y/o estético. En relación con la explotación de materias primas, los grupos humanos eligieron en mayor proporción la ortocuarcita del Grupo Sierras Bayas (GSB), mientras que otras rocas, como la ftanita, riolitas, metacuarcitas y los rodados costeros, fueron explotadas en menor medida. Esta tendencia prevalece incluso hasta el Holoceno tardío; sin embargo, para este lapso cronológico, se registró en algunos sectores un mayor uso de litologías de disponibilidad local (i.e., ftanita en el extremo noroccidental de Tandilia) (Messineo y Barros, 2015).

En relación con la forma de obtener los recursos líticos, se ha sugerido el empleo de dos estrategias (sensu Kuhn, 2004): el aprovisionamiento de los individuos y el aprovisionamiento de lugares. La primera estrategia habría sido empleada con continuidad desde el Holoceno temprano hasta el Holoceno tardío, mientras que la segunda se hace evidente en este último periodo. Esta última estrategia fue registrada en la cuenca media del río Quequén Grande, en la Ilanura Interserrana (Martínez y Mackie, 2003-2004), en sectores de esta llanura próximos al litoral atlántico (Bayón y Flegenheimer, 2004; Bonomo, 2005; Bayón et al., 2006) y en las lagunas del centro-oeste de la Pampa Húmeda (Messineo, 2011). Por otra parte, la presencia de litologías extraareales en algunos sitios ha sido interpretada como consecuencia de un incremento de las redes de interacción a larga distancia durante el Holoceno tardío (Bayón et al., 2006; Berón, 2006; Mazzanti, 2006; Messineo, 2011). Para este momento se observó una amplia variabilidad de artefactos y el empleo de una mayor diversidad de técnicas de manufactura, así como la incorporación del arco y la flecha, con lo cual se produjo una disminución en el tamaño y peso de las puntas de proyectil (Valverde y Martucci, 2004; Mazzanti, 2006).

En este contexto, el presente trabajo -basado en la tesis de licenciatura de la autora (Torino, 2018)- se propone contribuir al conocimiento del uso de los recursos líticos por parte de las sociedades cazadorasrecolectoras en el interior de la Ilanura Interserrana. Con este propósito, se llevó a cabo el análisis del conjunto lítico del sitio arqueológico superficial Las Toscas 3, ubicado en la margen noroeste de la laguna homónima, en el partido de Tres Arroyos.

\section{SITIO LAS TOSCAS 3}

La laguna Las Toscas se ubica a los $38^{\circ} 35^{\prime} \mathrm{S}$ y $59^{\circ} 46^{\prime} \mathrm{O}$, a unos $25 \mathrm{~km}$ de distancia del litoral atlántico (Figura 1). Debido a la abundancia y diversidad de materiales arqueológicos dispersos en sus márgenes, en el año 2007 comenzaron las investigaciones en este sector (Massigoge y Rafuse, 2013). Las tareas de campo permitieron la localización de tres sitios en superficie (Las Toscas 1, 2 y 3), uno en posición subsuperficial (Las Toscas 4) y otro en estratigrafía (Las Toscas 5; Figura 1).

El sitio Las Toscas 3 (TO3) se ubica en la margen noroeste de la laguna (Figura 1). Para la recolección sistemática de los materiales que se encontraban en superficie se realizaron seis transectas lineales que cubrieron una superficie aproximada de 6600 $\mathrm{m}^{2}$. Se recuperaron 1018 artefactos líticos, 13 restos faunísticos, 37 ecofactos, cuatro fragmentos de caracoles marinos, uno de cáscara de huevo y cuatro fragmentos de pigmentos minerales. La densidad del material lítico es de 0,16 artefacto/ $\mathrm{m}^{2}$. Debido a que los materiales aquí tratados son superficiales y no se cuenta con fechados radiocarbónicos, la variable temporal no puede ser tratada convenientemente más allá de estimaciones de grano grueso. No obstante, la información generada permite discutir algunas características del comportamiento tecnológico de los grupos cazadores-recolectores 


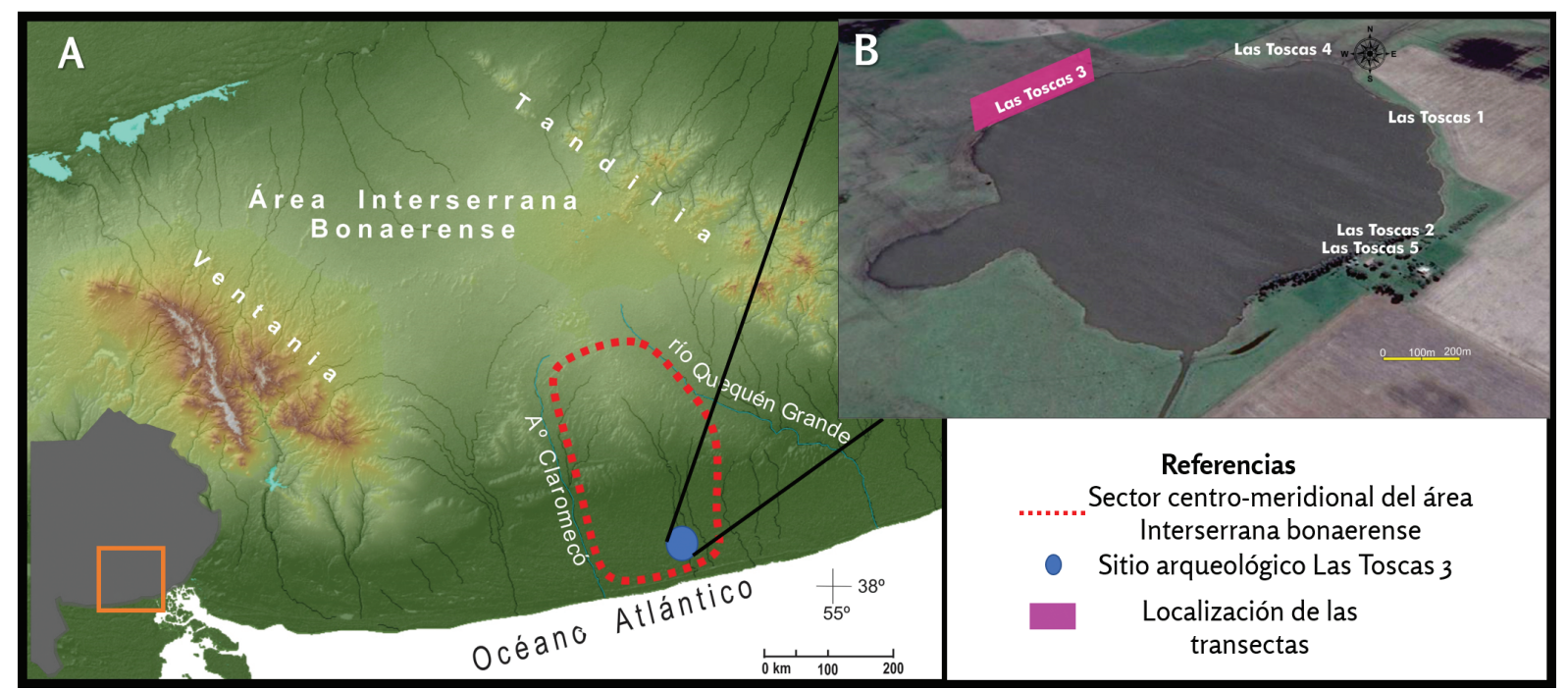

Figura 1. A. Localización del sitio arqueológico TO3 y área de estudio. B. Imagen satelital de Laguna Las Toscas.

que habitaron el área de estudio con respecto a los recursos líticos (i.e., obtención y transporte de materias primas, tendencias tecnológicas, entre otros). El hallazgo de una punta de proyectil triangular pequeña indica que al menos una parte de los eventos de ocupación de este sitio son asignables al Holoceno tardío, aunque es posible que existiesen ocupaciones previas (Politis y Madrid, 2001; Valverde y Martucci, 2004; Bonomo, 2005; Mazzanti, 2006; Pal y Messineo, 2014).

\section{METODOLOGÍA}

Los artefactos líticos fueron estudiados a nivel macroscópico. El análisis tecnomorfológico fue realizado siguiendo los lineamientos generales de la tipología de Aschero (1975, 1983). Además, se emplearon algunos criterios tomados de Boëda (1993), Inizan et al. (1995) y Aschero y Hocsman (2004) para el análisis de los instrumentos; de Paulides (2006) para los núcleos; y de Bellelli et al. (19851987) para los desechos de talla.

Para la distinción entre las ortocuarcitas de Ventania y Tandilia se siguieron principalmente los criterios macroscópicos definidos por Bayón y colaboradores (1999) y se realizaron comparaciones con muestras de mano procedentes de estos cordones serranos. Asimismo, la identificación del chert silíceo se realizó por comparación con muestras de mano de la Meseta del Fresco. Para discutir los planes de abastecimiento se emplearon las categorías definidas por Bayón y Flegenheimer (2004): rocas inmediatamente disponibles (localizadas hasta 10 $\mathrm{km}$ en línea recta desde del sitio); locales (hasta 60 km en línea recta); de distancia media (entre 60 y
100 km en línea recta) y de larga distancia (a más de $100 \mathrm{~km}$ en línea recta).

\section{RESULTADOS}

El conjunto analizado está compuesto por 1018 piezas, de las cuales el $82,2 \%$ corresponde a desechos de talla, el $17,5 \%$ a instrumentos y el $0,3 \%$ a núcleos. Se determinaron 14 tipos de materias primas, de las cuales la más frecuente es la ortocuarcita GSB, con el 85,5\%, seguida por la ftanita con el $8,8 \%$ y la metacuarcita con el 1,1\%. Las demás rocas se hallan representadas en una proporción igual o menor al 1\% (Tabla 1 ).

\section{Ortocuarcita GSB}

En el sitio se recuperaron tres núcleos de ortocuarcita GSB. Dos están enteros y el restante corresponde a un fragmento. Estas piezas carecen de corteza y presentan un avanzado estado de reducción, que impidió determinar la forma base. Uno de los núcleos enteros es de tipo prismático regular con lascados bidireccionales, de tamaño mediano pequeño, módulo longitud/anchura laminar normal y módulo anchura/espesor muy espeso. De las dos plataformas de percusión, una es plana y se encuentra agotada, mientras que la otra está totalmente agotada. El otro núcleo es bipolar, de tamaño mediano pequeño, módulo longitud/anchura laminar angosto y módulo anchura/espesor muy espeso. Su plataforma de percusión está agotada. En el caso del fragmento, no fue posible determinar a qué tipo de núcleo corresponde (Figura 2). 


\begin{tabular}{|c|c|c|c|c|c|c|c|c|}
\hline \multirow{2}{*}{$\begin{array}{c}\text { Categorías } \\
\text { artefactuales }\end{array}$} & & & & & & & \multirow{2}{*}{\multicolumn{2}{|c|}{ TOTAL }} \\
\hline & \multicolumn{2}{|c|}{ Desechos de talla } & \multicolumn{2}{|c|}{ Instrumentos } & \multicolumn{2}{|c|}{ Núcleos } & & \\
\hline Materias primas & $\mathrm{N}$ & $\%$ & $\mathrm{~N}$ & $\%$ & $\mathrm{~N}$ & $\%$ & $\mathrm{~N}$ & $\%$ \\
\hline Ortocuarcita GSB & 731 & 87,3 & 137 & 77 & 3 & 100 & 871 & 85,5 \\
\hline Ftanita & 60 & 7,2 & 30 & 17 & & & 90 & 8,8 \\
\hline Metacuarcita & 9 & 1,1 & 2 & 1,1 & & & 11 & 1,1 \\
\hline Dolomía silicificada & 7 & 0,8 & 2 & 1,1 & & & 9 & 0,9 \\
\hline Roca silícea & 6 & 0,7 & 4 & 2 & & & 10 & 1 \\
\hline Basalto & 7 & 0,8 & 1 & 0,6 & & & 8 & 0,8 \\
\hline Riolita & 1 & 0,1 & & & & & 1 & 0,1 \\
\hline ND & 1 & 0,1 & & & & & 1 & 0,1 \\
\hline Roca silícea & 4 & 0,5 & 1 & 6,6 & & & 5 & 0,5 \\
\hline Arenisca & 1 & 0,1 & 1 & 0,6 & & & 2 & 0,2 \\
\hline Cuarcita ND & 3 & 0,4 & & & & & 3 & 0,3 \\
\hline Roca granítica & 2 & 0,2 & & & & & 2 & 0,2 \\
\hline Indeterminada & 2 & 0,2 & & & & & 2 & 0,2 \\
\hline Chert silíceo & 1 & 0,1 & & & & & 1 & 0,1 \\
\hline Limolita silicificada & 2 & 0,2 & & & & & 2 & 0,2 \\
\hline Total & 837 & 82,2 & 178 & 17,5 & 3 & 0,3 & 1018 & 100 \\
\hline
\end{tabular}

Tabla 1. Clases artefactuales representadas por materia prima. Referencias: GSB: Grupo Sierras Bayas; ND: no determinada.

Se recuperaron 137 instrumentos, elaborados casi en su totalidad mediante la talla unifacial. La excepción es un instrumento modificado por uso (mano de molino). Entre los grupos tipológicos se destacan los instrumentos compuestos, conformados por diferentes grupos tipológicos: los filos en raedera y raspador son los más representados y se hallan en combinación con otros tipos de filos, como punta destacada, cuchillo, retoque sumario,

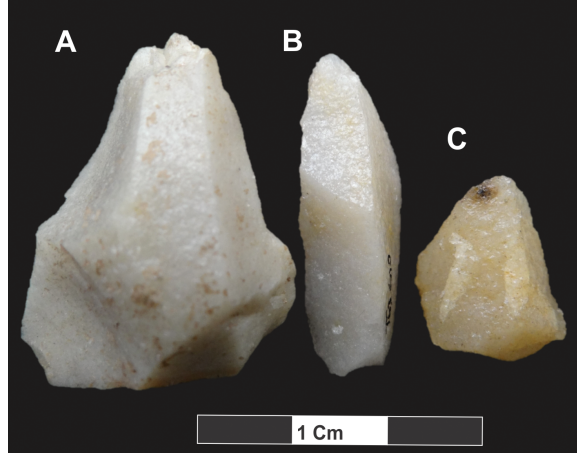

Figura 2. Núcleos de ortocuarcita GSB. Referencias: A- Núcleo prismático regular con lascados bidireccionales; B-Núcleo bipolar; C-Fragmento de núcleo.

raclette, pièce esquillée, muescas, entre otros (Figura 3). Asimismo, se observa una gran variabilidad de filos simples, como raederas, raspadores y lascas con mínima formatización. Otros grupos tipológicos se encuentran presentes en menores proporciones (Tabla 2). Se registró la presencia de corteza en solo un instrumento. En lo que respecta al estado, prevalecen los fragmentos de instrumentos $(70,6 \% ; n=96)$, seguidos por los enteros $(23,5 \% ; n=33)$ y las piezas fragmentadas $(5,9 \% ; n=8)$. Entre los instrumentos enteros, se observa una mayor representación de los tamaños mediano pequeño $(45,4 \% ; n=15)$ y mediano grande $(27,3 \% ; n=9)$, los módulos de longitud/anchura mediano normal $(36,3 \% ; n=12)$

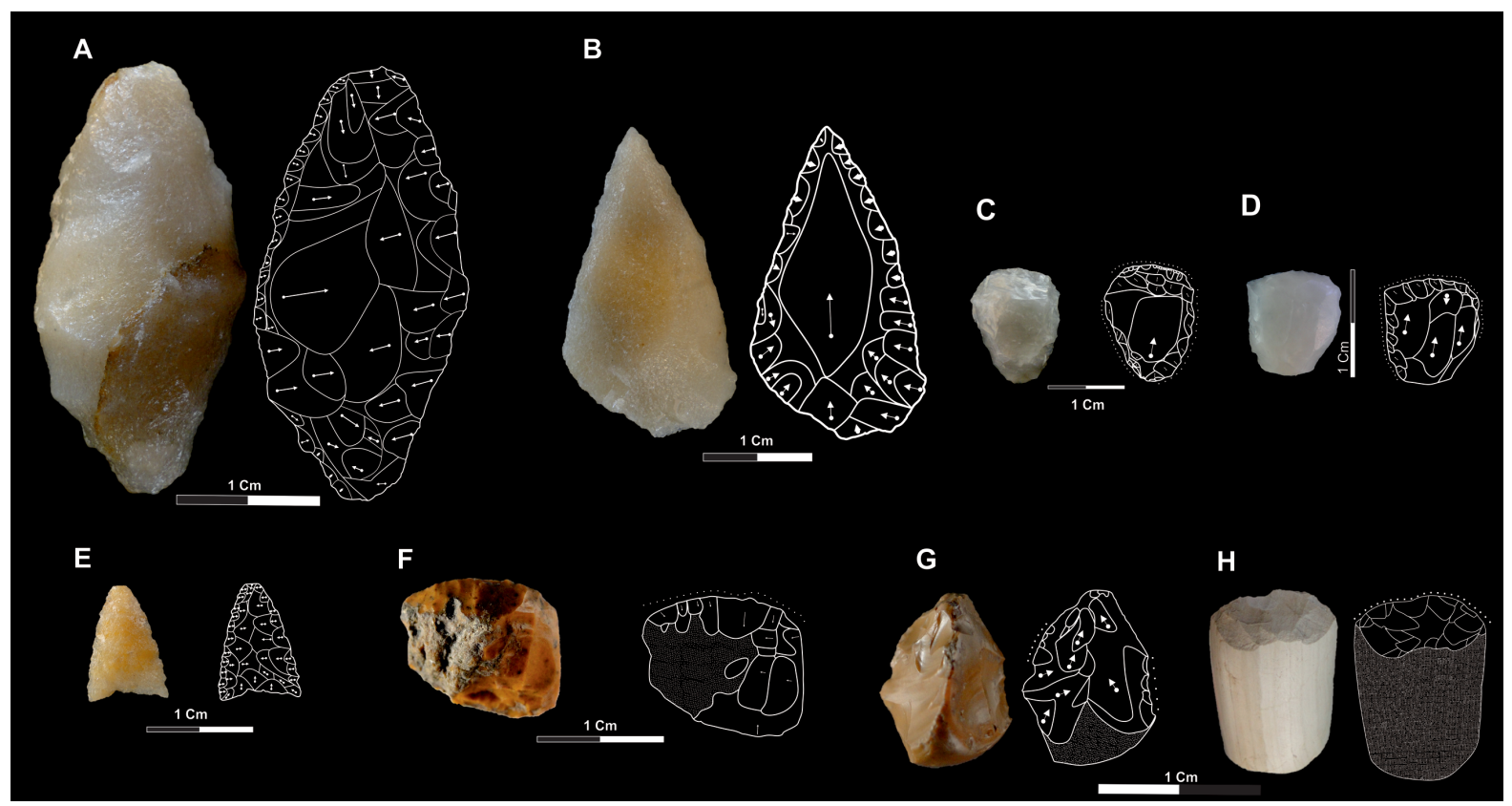

Figura 3. Artefactos formatizados del sitio TO3. Referencias: A. Instrumento compuesto ortocuarcita GSB; B. Raedera doble convergente en punta de ortocuarcita GSB; C. Raspador perimetral de ftanita; D. Raspador frontal de ftanita; E. Punta de proyectil triangular pequeña de ortocuarcita GSB; F. Raspador frontal de dolomía silicificada; G. Instrumento compuesto de sílice; H. Raspador frontal de sílice. 
Tecnología lítica en el sitio Las Toscas 3 (Ilanura Interserrana, región pampeana, Argentina)... Intersecciones en Antropología 21(1), enero-junio. 2020. ISSN-e 1850-373X

\begin{tabular}{|c|c|c|c|c|c|c|c|c|c|c|}
\hline \multirow[b]{2}{*}{ Grupo tipológico } & \multicolumn{8}{|c|}{ MATERIAS PRIMAS } & \multicolumn{2}{|c|}{ TOTAL } \\
\hline & $\begin{array}{c}\text { Ortocuarcita } \\
\text { GSB }\end{array}$ & Ftanita & Metacuarcita & $\begin{array}{c}\text { Dolomía } \\
\text { silicificada }\end{array}$ & $\begin{array}{c}\begin{array}{c}\text { Sílice } \\
\text { (rodado) }\end{array} \\
\end{array}$ & $\begin{array}{c}\text { Basalto } \\
\text { (rodado) }\end{array}$ & $\begin{array}{c}\text { Roca } \\
\text { silícea }\end{array}$ & Arenisca & $\mathrm{N}$ & $\%$ \\
\hline Instrumento compuesto & 40 & 6 & 1 & - & 1 & - & - & -- & 48 & 27 \\
\hline Raedera & 15 & - & 1 & - & - & - & - & - & 16 & 9 \\
\hline Raspador & 14 & 16 & - & 2 & 1 & - & 1 & - & 34 & 19,1 \\
\hline Pieza con retoque sumario & 12 & 1 & - & - & - & - & - & - & 13 & 7,3 \\
\hline $\begin{array}{l}\text { Lasca con rastros } \\
\text { complementarios }\end{array}$ & 8 & 2 & - & - & - & - & - & - & 10 & 5,7 \\
\hline Cuchillo & 3 & - & - & - & - & - & - & - & 3 & 1,7 \\
\hline Muesca & 2 & - & - & - & - & - & - & - & 2 & 1,1 \\
\hline Filo bisel asimétrico & 1 & - & - & - & - & - & - & - & 1 & 0,6 \\
\hline Punta de proyectil & 1 & - & - & - & - & - & - & - & 1 & 0,6 \\
\hline Pièce esquillée & 1 & - & - & - & 2 & 1 & - & - & 4 & 2,3 \\
\hline Perforador biconvexo & 1 & - & - & - & - & - & - & - & 1 & 0,6 \\
\hline Raclettes & 1 & 1 & - & - & - & - & - & - & 2 & 1,1 \\
\hline Artefacto modificado por uso & 1 & - & - & - & - & - & - & 1 & 2 & 1,1 \\
\hline Fragmento no determinado & 37 & 4 & - & - & - & - & - & - & 41 & 23 \\
\hline Total & 137 & 30 & 2 & 2 & 4 & 1 & 1 & 1 & 178 & 100 \\
\hline
\end{tabular}

Tabla 2. Grupos tipológicos por materia prima.

y mediano alargado $(21,2 \% ; n=7)$ y el módulo anchura/espesor espeso $(51,5 \% ; n=17)$. En cuanto a los soportes, el $88,5 \%$ corresponde a lascas angulares (Tabla 3). Con respecto a la serie técnica, la situación de los lascados indica que la reducción unifacial directa es la más representada, con el $86,8 \%(\mathrm{n}=119)$, seguida en menor medida por la reducción alterna $(6,5 \% ; n=9)$, la bifacial e inversa $(3 \% ; n=4$, cada una) y la alternante $(0,4 \% ; n=1)$. En general se observa el empleo del microrretoque y el retoque marginal para la elaboración de los instrumentos.

Con respecto a los desechos de talla $(87,3 \%$; $n=731$ ), prevalecen las lascas fracturadas con y sin talón, mientas que las enteras representan el 17,6\% $(n=129)$ del conjunto (Tabla 4$)$. Entre ellas predominan las de tamaño pequeño $(70,9 \% ; n=90)$ y módulo longitud/anchura mediano normal (36,2\%; $\mathrm{n}=46)$. En cuanto a los tipos de lascas, predominan las angulares $(78,8 \% ; \mathrm{n}=567)$. Con respecto al talón, el tipo más frecuente es el liso $(80 \% ; n=390)$.

\section{Ftanita}

Entre los instrumentos de ftanita $(17 \% ; \mathrm{n}=30)$ se destaca la alta frecuencia de raspadores (Figura 3) e instrumentos compuestos (Tabla 2); y entre estos últimos se identificaron diferentes subgrupos tipológicos: un filo raspador + filo raedera + filo raspador, un filo raspador + filo unifacial + filo con retoque sumario, dos filos pièce esquillée + un filo con rastros complementarios, entre otros. Con relación al estado, el $50 \%(n=15)$ se encuentra entero, el $43,3 \%(n=13)$ corresponde a fragmentos

\begin{tabular}{|c|c|c|c|c|c|c|c|c|c|c|}
\hline \multirow{2}{*}{$\begin{array}{l}\text { Forma base/ } \\
\text { Materia prima }\end{array}$} & \multirow{2}{*}{$\begin{array}{c}\text { Ortocuarcita } \\
\text { GSB }\end{array}$} & \multirow{2}{*}{ Ftanita } & \multirow{2}{*}{ Metacuarcita } & \multirow{2}{*}{$\begin{array}{c}\text { Dolomía } \\
\text { silicificada }\end{array}$} & \multirow{2}{*}{$\begin{array}{c}\text { Sílice } \\
\text { (rodado) }\end{array}$} & \multirow{2}{*}{$\begin{array}{l}\text { Basalto } \\
\text { (rodado) }\end{array}$} & \multirow{2}{*}{$\begin{array}{c}\text { Roca } \\
\text { silícea }\end{array}$} & \multirow{2}{*}{ Arenisca } & \multicolumn{2}{|c|}{ TOTAL } \\
\hline & & & & & & & & & $\mathrm{N}$ & $\%$ \\
\hline Lasca angular & 123 & 24 & 2 & 2 & & & 1 & & 152 & 85,4 \\
\hline Lasca de arista & 6 & 3 & - & - & - & - & - & - & 9 & 5,1 \\
\hline Lasca laminar & 1 & 1 & - & - & - & - & - & - & 2 & 1,1 \\
\hline Lasca secundaria & 1 & - & - & - & - & - & - & - & 1 & 0,6 \\
\hline Forma base no determinada & 1 & - & - & - & - & - & - & - & 1 & 0,6 \\
\hline Núcleo & 1 & 1 & - & - & 1 & - & - & - & 3 & 1,7 \\
\hline Artefacto formatizado retomado & 1 & - & - & - & - & - & - & - & 1 & 0,6 \\
\hline Guijarro & 1 & - & - & - & - & - & - & 1 & 2 & 1,1 \\
\hline No determinada por fractura & 2 & 1 & - & - & - & - & - & - & 3 & 1,7 \\
\hline Lasca primaria & - & - & - & - & 1 & 1 & - & - & 2 & 1,1 \\
\hline Lasca con dorso natural & - & - & - & - & 1 & - & - & - & 1 & 0,6 \\
\hline Rodado costero & - & - & - & - & 1 & & & - & 1 & 0,6 \\
\hline Total & 137 & 30 & 2 & 2 & 4 & 1 & 1 & 1 & 178 & 100 \\
\hline
\end{tabular}

Tabla 3. Formas base de instrumentos por materia prima. 


\begin{tabular}{|c|c|c|c|c|c|c|c|c|}
\hline & \multicolumn{6}{|c|}{ Materias primas } & \multicolumn{2}{|c|}{ Total } \\
\hline Estado $(\mathrm{N}=\mathbf{8 1 8})$ & $\begin{array}{c}\text { Ortocuarcita } \\
\text { GSB }\end{array}$ & Ftanita & Metacuarcita & $\begin{array}{l}\text { Dolomía } \\
\text { silicificada }\end{array}$ & $\begin{array}{c}\text { Sílice } \\
\text { (rodados) }\end{array}$ & $\begin{array}{c}\text { Basalto } \\
\text { (rodados) }\end{array}$ & $\mathrm{N}$ & $\%$ \\
\hline Lasca entera & 129 & 17 & 3 & 3 & 5 & 2 & 159 & 19,4 \\
\hline Lasca fracturada con talón & 358 & 26 & 5 & 2 & 1 & 1 & 393 & 48 \\
\hline Lasca fracturada sin talón & 233 & 15 & 1 & 2 & 1 & 1 & 253 & 31 \\
\hline Desecho no clasificable & 11 & 2 & & & & & 13 & 1,6 \\
\hline \multicolumn{9}{|l|}{ Tamaño $(\mathrm{N}=159)$} \\
\hline Pequeño & 16 & 3 & & & & 1 & 20 & 12,5 \\
\hline Mediano pequeño & 90 & 11 & 3 & 3 & 4 & & 111 & 70 \\
\hline Mediano grande & 22 & 3 & & & 1 & 1 & 27 & 17 \\
\hline Grande & 1 & & & & & & 1 & 0,5 \\
\hline \multicolumn{9}{|l|}{ Módulo $\mathrm{L} / \mathrm{A}(\mathrm{N}=159)$} \\
\hline Mediano normal & 47 & 6 & & 1 & 2 & 1 & 57 & 35,8 \\
\hline Corto ancho & 28 & 2 & & & & & 30 & 18,9 \\
\hline Corto muy ancho & 20 & 2 & & & & 1 & 23 & 14,5 \\
\hline Mediano alargado & 20 & 4 & 1 & & 1 & & 26 & 16,4 \\
\hline Laminar normal & 11 & 2 & 1 & 2 & & & 16 & 10 \\
\hline Corto anchísimo & 2 & & 1 & & & & 3 & 1,9 \\
\hline Laminar angosto & 1 & 1 & & & 2 & & 4 & 2,5 \\
\hline
\end{tabular}

Dentro de los desechos de talla $(\mathrm{n}=9)$ predominan las lascas angulares $(66,6 \% ; n=6)$. En su mayoría consisten en lascas fracturadas con talón $(55,5 \% ; n=5) y$ enteras (33,3\%; $\mathrm{n}=3$ ) y fracturada sin talón $(11,1 \% ; n=1)$. Las lascas enteras son de tamaño pequeño y de módulos de longitud/anchura:

y el $6,4 \%(n=2)$ se presenta fragmentado. Entre las piezas enteras, el $66,6 \%(n=10)$ es de tamaño pequeño, el $26,7 \%(n=4)$ mediano pequeño y el $6,6 \%(n=1)$, mediano grande. El módulo longitud/ anchura de todos los instrumentos enteros es corto ancho. Todos ellos fueron elaborados mayormente sobre lascas angulares mediante microrretoques y retoques en situación unifacial directa. Ninguno de los ítems presenta corteza.

Entre los desechos de talla $(7,2 \% ; n=60)$ predominan las lascas fracturadas. Las enteras representan el $28,3 \%(n=17)$ del conjunto. El $64,7 \%(n=11)$ de las lascas enteras son de tamaño pequeño y se observa una gran variabilidad en cuanto al módulo longitud/anchura (Tabla 4). En relación con los tipos de lascas, se hallan mejor representadas las angulares $(77,6 \% ; n=45)$. Los talones son, en su mayoría, lisos $(83,7 \%, \mathrm{n}=36)$.

\section{Metacuarcita}

Se identificaron dos instrumentos elaborados sobre metacuarcita: un instrumento compuesto entero y un fragmento de filo en raedera. El primero es de tamaño muy grande y módulo longitud/anchura mediano normal. Entre sus grupos tipológicos, se determinaron dos muescas y un cepillo. Estos fueron elaborados mediante retalla marginal y retoque marginal de manera alterna; mientras que el segundo artefacto fue confeccionado de manera unifacial directa por retoque marginal. Ambos instrumentos carecen de corteza. mediano alargado, corto ancho y laminar normal. Los talones son mayormente lisos $(66,6 \%$; $n=6)$.

\section{Dolomía silicificada}

Se recuperaron dos raspadores enteros confeccionados sobre lascas angulares (Figura 3). Ambos son de tamaño pequeño, módulo longitud/anchura corto ancho y corto muy ancho y módulo anchura/ espesor espeso. El filo del primer raspador se elaboró por retoques y microrretoques marginales sobre la cara dorsal de la lasca. En tanto que los filos del segundo se trabajaron de manera alterna, uno de ellos por retoque marginal, y el otro, por microrretoque marginal y retoque parcialmente extendido. Ninguno de los dos presenta corteza.

En cuanto a los desechos de talla $(\mathrm{n}=7)$, predominan las lascas enteras $(42,8 \% ; n=3)$, seguidas por las lascas fracturadas con y sin talón $(28,6 \%$; $\mathrm{n}=2$ para cada caso). La mayoría de las lascas son de tipo angular $(57,1 \% ; n=4)$ y bipolar $(42,8 \%$; $\mathrm{n}=3$ ). Las enteras son, en su mayoría, de tamaño mediano pequeño, módulo anchura/espesor poco espeso y presentan gran variabilidad en cuanto al módulo longitud/anchura. Los talones son, en su mayoría, lisos (42,8\%; $\mathrm{n}=3)$.

\section{Rodados costeros}

Dentro del grupo de los rodados costeros se identificaron tres tipos diferentes de materias primas: 
sílice $(n=10)$, basalto $(n=8)$ y riolita $(n=1)$. Entre las clases artefactuales presentes, en sílice se hallaron seis desechos y cuatro instrumentos; en basalto, siete desechos y un instrumento; y en riolita, un desecho. Además, se recuperó un desecho sobre un rodado costero de una roca que no pudo ser identificada. Dentro de los instrumentos de sílice se determinaron dos pièces esquillées, un instrumento compuesto (un filo bisel asimétrico + un filo en raspador) y un raspador (Figura 3). Los tres primeros grupos tipológicos se hallan enteros, mientras que el último es un fragmento. Las piezas enteras son de tamaño pequeño y mediano pequeño. En lo que respecta al módulo longitud/anchura, se encuentran representados el mediano normal, mediano alargado y laminar normal. Todos presentan un módulo anchura/espesor muy espeso. Se observa el empleo de diversos soportes (Tabla 3 ) sobre los que se elaboraron filos mediante microrretoque y retoques marginales de manera unifacial directa. El fragmento de raspador presenta un filo frontal elaborado mediante retoque marginal sobre la cara dorsal de una lasca primaria. Los desechos de sílice se encuentran mayormente enteros $(n=5)$, mientras que los dos restantes se hallan fracturados, con y sin talón. Las lascas enteras son de tamaño pequeño y presentan diferentes módulos de longitud/anchura (Tabla 4). En su totalidad corresponden a lascas primarias. Los talones son lisos y corticales.

En basalto se recuperó otra pièce esquillée entera. Esta es de tamaño mediano pequeño y módulo longitud/anchura mediano normal. Su forma base es un cuerpo central de un núcleo bipolar cuyo módulo anchura/espesor es espeso. Los filos se confeccionaron mediante microrretoque marginal. Entre los desechos de talla de basalto, se identificaron lascas fracturadas con talón $(57,1 \%$; $n=4)$, enteras $(28,6 \% ; n=2)$ y una fracturada sin talón. Las enteras son de tamaño mediano pequeño y módulo longitud/anchura corto muy ancho y mediano normal. En relación con el tipo, predominan las angulares $(\mathrm{n}=3)$. Los talones más representados son lisos. Por último, el desecho de riolita corresponde a una lasca bipolar entera de talón liso, la cual presenta corteza. Es de tamaño mediano pequeño y módulo longitud/anchura corto anchísimo.

\section{Materias primas escasamente representadas}

En muy baja frecuencia se identificaron artefactos de otras materias primas, entre las que se incluye arenisca (una lasca fracturada y un artefacto modificado por uso, el cual podría ser una mano de molino), roca granítica (dos lascas fracturadas), limolita silicificada (una lasca entera y una fracturada), chert silíceo (una lasca entera) y roca silícea (dos lascas fracturadas y una entera y un fragmento de filo en raspador).

\section{DISCUSIÓN}

El conjunto lítico de TO3 está integrado mayormente por desechos de talla y, en menor medida, instrumentos y núcleos. Sobre la base del análisis lítico, se identificaron un total de 14 tipos de materias primas representadas en diferentes frecuencias. En este sentido, se observa que se explotaron mayormente materias primas ubicadas a larga distancia, procedentes del sector centro-sur del sistema serrano de Tandilia (105 km) (Bayón et al., 1999; Bayón y Flegenheimer, 2004; Colombo, 2011). Los grupos habrían realizado movimientos hacia este sector, tanto residenciales como logísticos, para abastecerse principalmente de ortocuarcita del GSB $y$, de modo secundario, de ftanita. Mientras que, en el mismo sector, la dolomía silicificada y las rocas graníticas y silíceas pudieron ser obtenidas en el marco del abastecimiento de las rocas anteriormente mencionadas (embedded procurement, sensu Binford, 1979). En el caso de la ftanita, no se puede descartar la posibilidad de que una pequeña proporción de esta roca haya arribado desde los afloramientos y canteras-taller del sector noroccidental de Tandilia, que se ubican a $185 \mathrm{~km}$ de TO3 (Barros y Messineo, 2004).

De manera complementaria, se emplearon rodados costeros, recursos locales, cuyas fuentes se ubican en el litoral atlántico, a una distancia de 25 km desde el sitio (Bonomo, 2002, 2005). La presencia de rodados, así como de moluscos marinos en el sitio sugiere que los grupos cazadores-recolectores que ocuparon TO3 habrían visitado la costa en búsqueda de diversos recursos. El uso limitado de los rodados, a pesar de su carácter local, puede obedecer a que este tipo de materia prima no se adecúa a la manufactura de toda la gama de instrumentos que eran necesarios para llevar a cabo las distintas actividades, como sí lo hace la ortocuarcita GSB (Leipus, 2006; Pal, 2015).

En el sitio también se encuentran presentes, aunque en muy baja frecuencia, litologías extraareales, como son los casos de la riolita y metacuarcita, 
cuyas fuentes de aprovisionamiento se ubican en las sierras de Ventania (a más de 200 km) (Catella et al., 2010), o el chert silíceo, cuyos afloramientos se encuentran en el centro-oeste de la provincia de La Pampa $(600$ km) (Berón, 2006; Carrera Aizpitarte, 2007). Así como ha sido observado en otros sitios de la llanura Interserrana y del sistema serrano de Tandilia, estas rocas parecen haber ingresado mayormente como instrumentos terminados, lo cual permite proponer que habrían sido obtenidas a través de redes de interacción con otros grupos que ocupaban diferentes territorios de la región pampeana Esta situación podría estar vinculada al incremento de las redes de interacción a larga distancia hacia finales del Holoceno (Bayón et al., 2006; Mazzanti, 2006; Messineo, 2011).

La ortocuarcita GSB habría ingresado al sitio bajo la forma de núcleos, soportes (e.g., lascas espesas y muy espesas), preformas e instrumentos. Las primeras etapas de la cadena operativa, vinculadas al descortezamiento, reducción inicial de la roca y preparación para el transporte, se habrían llevado a cabo en el área de canteras (Paulides, 2006, 2007; Colombo, 2011). Esto se ve reflejado en el pequeño tamaño de los núcleos recuperados en el sitio -los cuales, además, se encuentran agotados-, en la escasa frecuencia de desechos de talla de tamaños grandes $y$, fundamentalmente, en el bajo índice de productos de talla con remanentes de corteza. Una vez en el sitio, esta roca se utilizó con diferentes fines: se destaca la confección de soportes de distintos módulos de longitud/anchura y espesor, que habrían sido utilizados para elaborar una gran variedad de grupos tipológicos, principalmente instrumentos compuestos, raederas y raspadores (Figura 4).

En cuanto a la ftanita, la ausencia de núcleos y la mayor proporción de instrumentos en relación con los desechos de talla sugieren que esta pudo haber sido transportada principalmente como formas base o artefactos formatizados. En el sitio, lascas de diferentes módulos de longitud/anchura y espesor fueron usadas como soportes para confeccionar distintos artefactos (e.g., raspadores, lascas con rastros complementarios, instrumentos compuestos). Sin embargo, el uso de esta roca estuvo dirigido principalmente a la confección de raspadores, lo cual, como señalaron otros investigadores, podría estar relacionado con una mejor adecuación de esta materia prima para este fin y con las propiedades mecánicas de esta roca y de los filos para ejercer una actividad determinada (Figura 4) (Bayón et al., 2006; Pal, 2009, 2015; Barros et al., 2015).

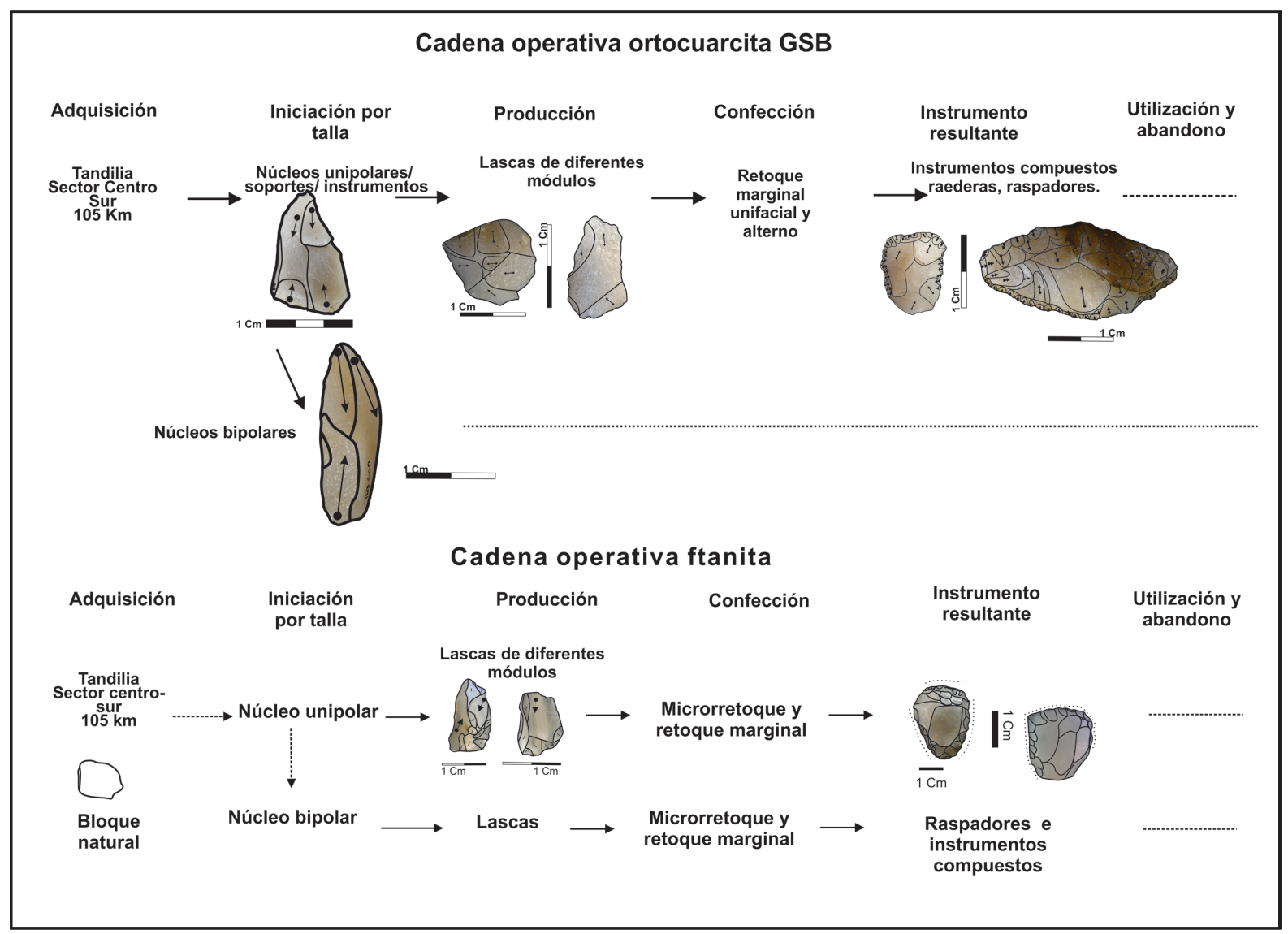

Figura 4. Cadenas operativas de ortocuarcita GSB y ftanita. 
En relación con los rodados, se observa que habrían arribado en modos diversos, ya sea en su forma original o como instrumentos ya finalizados. Por último, las materias primas escasamente representadas (dolomía silicificada, arenisca, metacuarcita, riolita, limolita silicificada, rocas graníticas y silíceas y chert silíceo) podrían haber ingresado bajo la forma de instrumentos que pudieron ser utilizados y acondicionados en TO3.

Respecto de las técnicas de talla, para la mayoría de las materias primas se observa un predominio de la talla unipolar. El empleo de la talla bipolar fue detectado en muy baja proporción, preferentemente sobre ortocuarcita GSB, ftanita y rodados costeros. Con respecto a las dos primeras rocas, esto se ve reflejado tanto en algunos desechos de talla como en la presencia de núcleos que fueron reducidos mediante esta técnica. Esta evidencia, en conjunto con el empleo de núcleos bipolares agotados como forma base de artefactos y la alta proporción de instrumentos compuestos, permite plantear la intención de maximizar el uso de estas materias primas, de buena calidad para la talla pero disponibles a larga distancia. En el caso de los rodados costeros, la talla bipolar se empleó para su reducción, la obtención de soportes y la manufactura de artefactos, situación que fue asociada a la reducción exitosa de pequeños nódulos de rocas duras y/o redondeadas, como es el caso de los rodados costeros (Flegenheimer et al., 1995; Curtoni, 1996).

El conjunto lítico de Las Toscas 3 presenta semejanzas con otros sitios del área de estudio que han sido ocupados a lo largo del Holoceno (e.g., Las Brusquillas 1, 2, 3 y 4, Las Toscas 5, Cortaderas, San Luis y Laguna La Vizcacha). En todos los conjuntos, se destaca la utilización preferencial de la ortocuarcita GSB y el uso secundario de otras litologías, como la ftanita. Los rodados costeros no siempre están presentes y, cuando lo están, se registran en frecuencias muy bajas (Massigoge, 2007, 2011a, 2011b, 2012; Massigoge y Pal, 2011; Massigoge et al., 2014, 2018; Torino, 2018). Esta situación se repite en la gran mayoría de los sitios del interior de la Ilanura Interserrana con cronologías desde el Holoceno temprano al tardío (e.g., Arroyo Seco 2, Paso Otero 3 y 4, El Guanaco, Tres Reyes 1, Zanjón Seco 2, Laguna La Barrancosa y El Guanaco 1 y sitio en superficie, entre otros) (Bayón y Flegenheimer, 2004; Madrid et al., 2002; Messineo, 2008). La importancia de la ortocuarcita GSB solo se reduce considerablemente en algunos sitios que se encuentran cercanos a otras fuentes potenciales de materias primas (e.g., costa atlántica, sistemas serranos de Ventania y Tandilia). En estos, se observa una mayor frecuencia de los recursos líticos locales (Politis y Madrid, 2001; Barros y Messineo, 2004; Bonomo, 2005; Catella, 2014; Mazzanti et al., 2015; Donadei, 2017, entre otros).

En TO3, así como en otros sitios del área de estudio, se registró el ingreso de las rocas en un estado avanzado de reducción, por lo que solo es posible registrar las etapas intermedias y finales de las cadenas operativas. La preparación inicial de la ortocuarcita GSB y la ftanita para su transporte y utilización en otros espacios ocupados se habría realizado en las canteras. Los talladores habrían partido de estos lugares con núcleos preparados, formas base e instrumentos en distintas etapas de confección (Barros, 2011; Colombo, 2011; Paulides, 2006, 2007). Este conjunto artefactual habría constituido el equipo personal que los talladores trasladaban con ellos. Hasta el momento, en los sitios del área de estudio no se ha observado la presencia de núcleos grandes que indicaran una estrategia de aprovisionamiento de los lugares con materias primas, lo cual sí se ha identificado en lugares próximos, como la localidad El Guanaco, la cuenca media del río Quequén Grande o algunos sectores del litoral atlántico (Martínez y Mackie, 2003-2004; Bayón y Flegenheimer, 2004; Bonomo, 2005; Bayón et al., 2006). Esta situación podría deberse a un uso diferencial de estos distintos espacios o a un sesgo cronológico de los conjuntos analizados (Massigoge y Pal, 2011; Massigoge et al., 2018).

\section{CONCLUSIÓN}

En términos generales, en TO3 se observa el empleo de diversas estrategias tecnológicas en relación con la gestión de los recursos líticos. El conjunto analizado sugiere que las primeras etapas de las cadenas operativas se desarrollaron en otros sectores del paisaje, debido a que en el sitio se encuentran mayormente representadas las etapas intermedias y finales de las secuencias, tanto en ortocuarcita GSB como en las restantes materias primas.

El análisis de los materiales líticos de TO3, en conjunto con la información generada para otros sitios de la Ilanura Interserrana permite caracterizar las estrategias tecnológicas implementadas por las 
sociedades cazadoras-recolectoras. En este sentido, no se observan grandes cambios en la tecnología lítica a lo largo del Holoceno. Esto se ve evidenciado, por un lado, en el patrón de selección y explotación de los recursos líticos, que exhibe modos de aprovisionamiento diferentes para cada una de las materias primas: directo para las rocas que provienen del sistema serrano de Tandilia y los rodados costeros, e indirecto para aquellas litologías que se encuentran representadas en baja proporción y que provienen de fuentes extraareales; por otro lado, en los métodos y técnicas de talla empleados para la confección de determinados tipos de instrumentos. Se registró una marcada tendencia al empleo de la talla unifacial, principalmente por percusión directa. La talla bipolar fue empleada mayormente dentro de una estrategia de maximización de las materias primas, como es el caso de los núcleos de ortocuarcita GSB.

El registro arqueológico de Las Toscas 3 es el resultado de la acción de distintos procesos, tanto culturales como naturales. En este sentido, este contexto puede ser considerado de muy baja integridad y resolución temporal (sensu Binford, 1981). Debido a la pérdida de información espacial, a la posibilidad de mezcla de materiales de distinto origen y a la deficiente preservación de algunas clases de evidencias materiales (e.g., restos faunísticos, carbón), resulta dificultoso realizar inferencias detalladas sobre su funcionalidad. Sin embargo, la información generada a partir de este análisis permitió discutir algunas características relativas al uso de los recursos líticos dado por las sociedades cazadoras-recolectoras en el interior de la llanura Interserrana a lo largo del Holoceno.

\section{Agradecimientos}

Quiero agradecer a Agustina Massigoge y a Paula Barros por su confianza y por su apoyo incondicional. A Manuel Carrera Aizpitarte por haber leído el manuscrito y sus valiosos comentarios. A los evaluadores/as del trabajo por sus exhaustivos comentarios que lo mejoraron sustancialmente. Este trabajo se llevó a cabo con recursos provenientes de subsidios de investigación otorgados por la ANPCyT (PICT 2010-0235, PICT 2013-0199 y PICT 20152777). Todo lo aquí escrito es pura responsabilidad de la autora.

\section{REFERENCIAS CITADAS}

Aldazábal, V. y Eugenio, E. (2010). El sector costero entre Punta Rasa y Faro Querandí (Buenos Aires) como potencial fuente de recursos líticos de cazadoresrecolectores. En M. Berón, M. Bonomo, C. Montalvo, C. Aranda, L. Luna y M. Carrera Aizpitarte (Eds.), Mamül Mapu: pasado y presente desde la arqueología pampeana, Vol. II (pp. 175-189). Ediciones del Espinillo.

Aschero, C. (1975). Ensayo para una clasificación morfológica de artefactos líticos. Informe presentado al CONICET [Manuscrito inédito]. Copia disponible en el Laboratorio № 3, División Científica de Arqueología, Museo de La Plata, Universidad Nacional de La Plata.

Aschero, C. (1983). Ensayo para una clasificación morfológica de artefactos líticos. Revisión [Manuscrito inédito]. Guías de Cátedra. Ergología y Tecnología. Facultad de Filosofía y Letras. Universidad de Buenos Aires.

Aschero, C. y Hocsman, S. (2004). Revisando cuestiones tipológicas en torno a la clasificación de artefactos bifaciales. En M. Ramos, A. Acosta y D. Loponte (Eds.), Temas de Arqueología. Análisis lítico (pp. 7-25). Universidad Nacional de Luján.

Barros, M. P. (2011). El uso del espacio del área Interserrana durante el Pleistoceno final/Holoceno. Su análisis a partir de la representación y el modo de explotación de las materias primas líticas. CazadoresRecolectores del Cono Sur, 5, 37-57.

Barros, M. P. y Messineo, P. G. (2004). Identificación y aprovisionamiento de chert o ftanita en la cuenca superior del arroyo Tapalqué. Estudios Atacameños, 28, 87-103.

Barros, M. P., Messineo, P. G. y Colantonio, M. J. (2015). Chert quarries and workshops in the Humid Pampa sub-region: New contributions on exploitation techniques and circulation through study of chaînes opératoires. Quaternary International, 375, 99-112.

Bayón, C. y Flegenheimer, N. (2004). Cambio de planes a través del tiempo para el traslado de roca en la pampa bonaerense. Estudios Atacameños, 28, 59-70.

Bayón, C. y Zavala, C. (1997). Coastal sites in south Buenos Aires a review of Piedras Quebradas. En J. Rabassa y M. Salemme (Eds.), Quaternary of South America and Antartic Peninsula, Vol.10 (pp. 229-253). Brookfield.

Bayón, C., Flegenheimer, N., Valente, M. y Pupio, A. 1999 Dime cómo eres y te diré de dónde vienes:

La procedencia de rocas cuarcíticas en la región pampeana. Relaciones de la Sociedad Argentina de Antropología, XXIV, 187-235. 
Bayón, C., Flegenheimer, N., y Pupio, A. (2006). Planes sociales en el abastecimiento y traslado de roca en la Pampa Bonaerense en el Holoceno Temprano y Tardío. Relaciones de la Sociedad Argentina de Antropología, XXXI, 19-45.

Bellelli, C., Guráieb, A. G. y García, J. A. (1985-1987). Propuesta para el análisis y procesamiento por computadora de desechos de talla lítica (DELCO Desechos de talla líticos computarizados). Arqueología Contemporánea, 2(1), 36-53.

Berón, M. (2006). Relaciones interétnicas e identidad social en el registro arqueológico. En V. Williams y B. Alberti (Eds.), Género y etnicidad en la arqueología sudamericana (pp. 119-138). Facultad de Ciencias Sociales, Universidad Nacional del Centro de la Provincia de Buenos Aires.

Binford, L. (1979). Organization and formation processes: looking at curated technologies. Journal of Anthropological Research, 35(3), 255-273.

Binford, L. (1981). Behavioral Archaeology and the "Pompeii Premise". Journal of Anthropological Research 37 (3), 195-208.

Böeda, E. (1993). Le débitage discoïde et le débitage Levallois récurrent centripède. Bulletin de la Société Préhistorique Française, 90(6), 392-404.

Bonomo, M. (2002). Distribución espacial y tecnología en el litoral marítimo bonaerense. En D. Mazzanti, M. Berón y F. Oliva (Eds.), Del Mar a los Salitrales. Diez mil años de Historia Pampeana en el Umbral del Tercer Milenio (pp. 185-204). Laboratorio de Arqueología, Facultad de Humanidades, Universidad Nacional de Mar del Plata.

Bonomo, M. (2005). Costeando las Ilanuras. Arqueología del litoral marítimo pampeano. Sociedad Argentina de Antropología (Colección Tesis Doctorales).

Carrera Aizpitarte, M. (2007). Estrategias de aprovisionamiento lítico en el área centro-este de la provincia de La Pampa [tesis de licenciatura inédita, Universidad Nacional del Centro de la Provincia de Buenos Aires].

Catella, L., Moirano, J. y Oliva, F. (2010). Disponibilidad de materias primas líticas y su importancia para el análisis del uso del espacio y la organización de la tecnología en sociedades cazadoras recolectoras. En M. Berón, L. Luna, M. Bonomo, C. Montalvo, C. Aranda y M. Carrera Aizpitarte (Eds.), Mamül Mapu: pasado y presente desde la arqueología pampeana, Vol. II (pp. 239-253). Ediciones del Espinillo.

Colombo, M. (2011). El área de abastecimiento de las ortocuarcitas del grupo Sierras Bayas y las posibles técnicas para su obtención entre los cazadores y recolectores pampeanos. Intersecciones en Antropología, 12, 155-166.

Curtoni, R. P. (1996). Experimentando con bipolares: indicadores e implicancias arqueológicas. Relaciones de la Sociedad Argentina de Antropología, XXI, 187-214.

Donadei, J. P. (2017). Gestión de la materia prima y estrategias de talla durante el Holoceno medio en Tandilia Oriental: el caso de Cueva Tixi (Buenos aires, Argentina). Comechingonia, 21(2), 379-399.

Flegenheimer, N. (1991). La liebre un sitio canterataller. Boletín del Centro de Registro del Patrimonio Arqueológico y Paleontológico, 2, 58-64.

Flegenheimer, N., Bayón, C. y González De Bonaveri, M. I. (1995). Técnica simple, comportamientos complejos: La talla bipolar en la arqueología bonaerense. Relaciones de la Sociedad Argentina de Antropología, XX, 81-110.

Flegenheimer, N., Kain, S., Zárate, M. y Barna, A. (1996). Aprovisionamiento de rocas cuarcíticas en Tandilia. Las canteras del Arroyo Diamante. Arqueología, 6, 117-143.

Flegenheimer, N., Zárate, M. y Valente, M. (1999). El área de canteras Arroyo Diamante, Barker, Sierras de Tandil. Actas XII Congreso Nacional de Arqueología Argentina, t. III, 134-138.

Inizan M., Reduron, M., Roche, H. y Tixier, J. (1995). Techologie de la Pierre Taillée. CREP.

Kuhn, S. (2004). Upper Paleolithic raw material economies at Ücagızlı cave, Turkey. Journal of Anthropological Archaeology, 23, 431-448.

Leipus, M. (2006). Análisis de los modos de uso prehispánicos de las materias primas líticas en el Sudeste de la Región Pampeana. Una aproximación funcional [tesis doctoral inédita, Universidad Nacional de La Plata].

Madrid, P., Politis, G., March, R. y Bonomo, M. (2002). Arqueología microrregional en el sudeste de la Región Pampeana Argentina: el curso del río Quequén Salado. Relaciones de la Sociedad Argentina de Antropología, XXVII, 327-355.

Martínez, G. y Mackie, Q. (2003-2004). Late Holocene human occupation of the Quequén Grande River valley botton: settlement systems end an example of a built environment in the Argentine Pampa. En L. Barham (Ed.), The Archaeology and anthropology of huntergatherers (pp. 78-202). Briston.

Massigoge, A. (2007). Resultados preliminares de las investigaciones arqueológicas desarrolladas en el partido de San Cayetano (provincia de Buenos Aires). En C. Bayón, A. Pupio, M. I. González, N. Flegenheimer y M. Frère (Eds.), Arqueología en las Pampas, t. 2 (pp. 511-534). Sociedad Argentina de Antropología. 
Massigoge, A. (2011a). Nuevas evidencias arqueológicas del Holoceno tardío en el Área Interserrana: el sitio Las Brusquillas 2 (partido de San Cayetano, provincia de Buenos Aires, Argentina). Cazadores-Recolectores del Cono Sur, 5, 179-195.

Massigoge, A. (2011b). Nuevas evidencias arqueológicas del Holoceno medio y tardío del área Interserrana: localidad arqueológica Las Brusquillas (partido de San Cayetano, provincia de Buenos Aires). Revista del Museo de La Plata. Arqueología, 12(86), 55.

Massigoge, A. (2012). Las Brusquillas 1 (partido de San Cayetano, provincia de Buenos Aires): un nuevo sitio del Holoceno tardío del área Interserrana de la región pampeana. Intersecciones en Antropología, 13, 377-392.

Massigoge, A. y Pal, N. (2011). Producción y uso de artefactos líticos en contextos cazadores-recolectores del Holoceno tardío del área Interserrana (Argentina): análisis integral de la diversidad tecno-morfológica y funcional. Revista Española de Antropología Americana, 41(1), 51-73.

Massigoge, A. y Rafuse, D. (2013). Investigaciones arqueológicas en la localidad Laguna Las Toscas (partido de Tres Arroyos, provincia de Buenos Aires). XVIII Congreso Nacional de Arqueología Argentina (pp. 550-551). INCIHUSA-CONICET/Universidad Nacional de La Rioja.

Massigoge, A., Rodríguez, J. M., Favier Dubois, C. M., Rafuse, D. J., Steffan, P. G., Rodríguez, M. N. y Torino, R. F. (2014). Sitio Las Toscas 5: un nuevo contexto arqueológico del Holoceno medio en el área Interserrana de la región pampeana (Argentina). Libro de resúmenes del VII CARPA [Publicación digital en $\mathrm{CD}]$.

Massigoge, A., Rodríguez, M., Rafuse, D., Torino, R., Favier Dubois, C. y Steffan, P. (2018). Investigaciones arqueológicas en el sitio Las Brusquillas 3 (Holoceno Tardío, Región Pampeana, Argentina). Revista Arqueología, 24(1), 147-171.

Mazzanti, D. (2006). La constitución de territorios sociales durante el Holoceno tardío. El caso de las sierras de Tandilia, Argentina. Relaciones de la Sociedad Argentina de Antropología, XXXI, 277-300.

Mazzanti, D., Martínez, G., y Quintana, C. (2015). Asentamientos del Holoceno medio en Tandilia oriental. Aportes para el conocimiento de la dinámica poblacional de la región pampeana, Argentina. Relaciones de la Sociedad Argentina de Antropología, XL, 209-231.

Messineo, P. G. (2008). Investigaciones arqueológicas en la cuenca superior del arroyo Tapalqué (partidos de
Olavarría y Benito Juárez, provincia de Buenos Aires) [tesis doctoral inédita, Universidad de La Plata].

Messineo, P. G. (2011). Investigaciones arqueológicas en la cuenca superior del Arroyo Tapalqué. Un modelo de ocupación humana para el centro de la subregión Pampa Húmeda durante el Holoceno tardío. Intersecciones en Antropología, 12, 275-291.

Messineo, P. G. y Barros, M. P. (2015). Lithic raw materials and modes of exploitation in quarries and workshops from the center of the Pampean grassland of Argentina. Lithic Technology, 40(I), 3-20.

Messineo, P. G., Barros, M. P., Poiré, D. G. y Gómez Peral, L. (2004). Características litológicas de los niveles de chert o ftanitas en las Sierras Bayas (Pdo. de Olavarría, Pcia. de Buenos Aires). En G. Martínez, M. Gutiérrez, R. Curtoni, M. Berón y P. Madrid (Eds.), Aproximaciones contemporáneas a la arqueología pampeana. Perspectivas teóricas, metodológicas, analíticas y casos de estudio (pp. 307-319). Facultad de Ciencias Sociales, Universidad Nacional del Centro de la Provincia de Buenos Aires.

Oliva, F. y Moirano, J. (1997). Primer informe sobre aprovisionamiento primario riolita en Sierra de la Ventana (Pcia. Buenos Aires, Argentina). En M. Berón y G. Politis (Eds.), Arqueología Pampeana en la década de los '90, pp. 137-146. Museo de Historia Natural de San Rafael; INCUAPA-UNCPBA.

Pal, N. (2009). Diseños y usos de los artefactos líticos manufacturados por talla en la cuenca superior del Arroyo Tapalqué (provincia de Buenos Aires). Comechingonia, 17, 171-187.

Pal, N. (2015). Estrategias de uso de instrumentos líticos en la cuenca superior del arroyo Tapalqué durante el Holoceno tardío (provincia de Buenos Aires). Intersecciones en Antropología, 16, 53-68.

Pal, N. y Messineo, P. G. (2014). Aportes a la interpretación de las actividades llevadas a cabo en sitios superficiales a partir del análisis funcional. Revista del Museo de Antropología, 7(1), 79-92.

Paulides, L. (2006). El núcleo de la cuestión. El análisis de los núcleos en los conjuntos líticos. En C. Pérez de Micou (Ed.), El modo de hacer las cosas. Artefactos y ecofactos en arqueología (pp. 67-97). Facultad de Filosofía y Letras, Universidad Nacional de Buenos Aires.

Paulides, L. (2007). Prepárate, vas a viajar: sobre tendencias productivas en la formatización de núcleos en el complejo de canteras de Arroyo Diamante, partido de Benito Juárez (provincia de Buenos Aires). En C. Bayón, A. Pupio, M. I. González, N. Flegenheimer y M. Frère (Eds.), Arqueología en las Pampas, t. I (pp. 233-249). Sociedad Argentina de Antropología. 
Tecnología lítica en el sitio Las Toscas 3 (Ilanura Interserrana, región pampeana, Argentina)... Intersecciones en Antropología 21(1), enero-junio. 2020. ISSN-e 1850-373X

Politis, G. y Madrid, P. (2001). Arqueología pampeana: estado actual y perspectivas. En E. Berberián y A. Nielsen (Eds.), Historia Argentina Prehispánica, t. 2 (pp. 737-814). Brujas.

Torino, R. (2018). Modos de abastecimiento y circulación de rocas durante el Holoceno tardío en el sector centro-meridional del área Interserrana bonaerense (sudeste pampeano) [tesis de licenciatura inédita, Universidad Nacional del Centro de la Provincia de Buenos Aires].
Valverde, F. y Martucci, M. (2004). Estudio tecno-tipológico de las puntas de proyectil del sitio Cueva El Abra (Provincia de Buenos Aires). En G. Martínez, M. A. Gutiérrez, R. Curtoni, M. Berón y P. Madrid (Eds.), Aproximaciones Contemporáneas a la Arqueología Pampeana. Perspectivas Teóricas, Metodológicas, Analíticas y Casos de Aplicación (pp. 419-434). Facultad de Ciencias Sociales, Universidad Nacional del Centro de la Provincia de Buenos Aires. 
